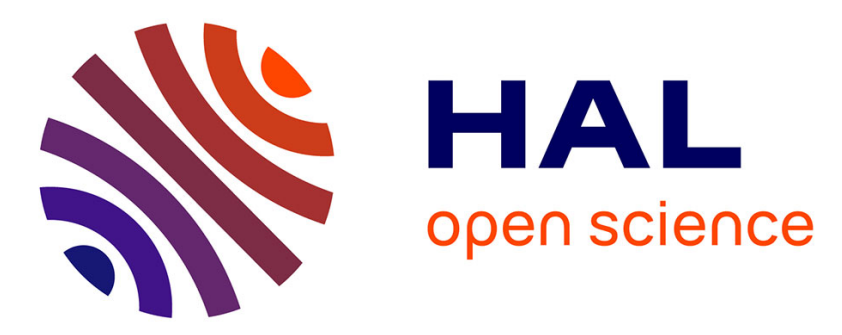

\title{
Detecting Protein-Protein Interactions In Vivo with FRET using Multiphoton Fluorescence Lifetime Imaging Microscopy (FLIM)
}

\author{
David Llères, Samuel Swift, Angus I Lamond
}

\section{- To cite this version:}

David Llères, Samuel Swift, Angus I Lamond. Detecting Protein-Protein Interactions In Vivo with FRET using Multiphoton Fluorescence Lifetime Imaging Microscopy (FLIM). Current Protocols in Cytometry, 2007, 42 (1), 10.1002/0471142956.cy1210s42 . hal-03027047

\section{HAL Id: hal-03027047 https://hal.science/hal-03027047}

Submitted on 26 Nov 2020

HAL is a multi-disciplinary open access archive for the deposit and dissemination of scientific research documents, whether they are published or not. The documents may come from teaching and research institutions in France or abroad, or from public or private research centers.
L'archive ouverte pluridisciplinaire HAL, est destinée au dépôt et à la diffusion de documents scientifiques de niveau recherche, publiés ou non, émanant des établissements d'enseignement et de recherche français ou étrangers, des laboratoires publics ou privés. 


\title{
Detecting Protein-Protein Interactions In Vivo with FRET using Multiphoton Fluorescence Lifetime Imaging Microscopy (FLIM)
}

\author{
David Llères, ${ }^{1}$ Samuel Swift, ${ }^{1}$ and Angus I. Lamond ${ }^{1}$ \\ ${ }^{1}$ Wellcome Trust Biocentre, College of Life Sciences, University of Dundee, \\ United Kingdom
}

\begin{abstract}
Protein interactions are critical for many processes in mammalian cells. Such interactions include the stable association of proteins within multi-subunit complexes and the transient association of regulatory proteins. Information about protein interactions in cells has previously come from either in vitro analyses using recombinant expressed proteins, or from yeast 2-hybrid studies. A limitation of this approach is that the protein interaction is studied in isolation, without regard to the many competing protein interactions that can occur within cells. This unit presents a light microscopy approach for detecting proteinprotein interactions in vivo based on the measurement of FRET using the multiphoton fluorescence lifetime imaging microscopy (FLIM) technique. By using the FLIM-FRET technique, the spatial organization and quantification of such interactions in a living cell can be characterized. A detailed protocol describing the complete microscope procedure and the choice of the appropriate experimental controls as well as the FRET calculations is also included. Curr. Protoc. Cytom. 42:12.10.1-12.10.19. (C) 2007 by John Wiley \& Sons, Inc.
\end{abstract}

Keywords: fluorescence lifetime $\bullet$ GFP $\bullet$ mCherry $\bullet$ FRET $\bullet$ FLIM $\bullet$ multiphoton $\bullet$ protein interaction

\section{INTRODUCTION}

This unit presents a protocol for detecting molecular interactions between two proteins at a specific location within mammalian cells. The assay is based on fluorescence lifetime imaging (FLIM) by using time-correlated single-photon counting (TCSPC; Lakowicz, 1999) to measure fluorescence resonance energy transfer (FRET) between two proteins. To achieve this, each protein must carry a separate fluorophore that together constitute a FRET pair.

In fluorescence microscopy, fluorophores targeted to cellular structures of interest are irradiated with high-intensity light. When these molecules absorb a quantum of light, a valence electron is boosted up into a higher energy orbit, creating an excited state (Fig. 12.10.1). When this electron returns to its original, lower energy orbit (the ground state level), a quantum of light may be emitted. This occurs on a nanosecond timescale. Fluorescence is thus a relaxation process that offers a pathway for molecules to relax from an excited state back to a nonexcited state (Fig. 12.10.1). However, fluorescence is only one of a number of possible de-excitation pathways available to electrons. Vibrational relaxation is probably the most common pathway, which leads to the loss of some energy prior to the emission of light. The loss of energy through vibrational relaxation means that less energy is available for emission as fluorescence (Lakowicz, 1999). Because wavelength varies inversely to radiative energy, fluorescence 


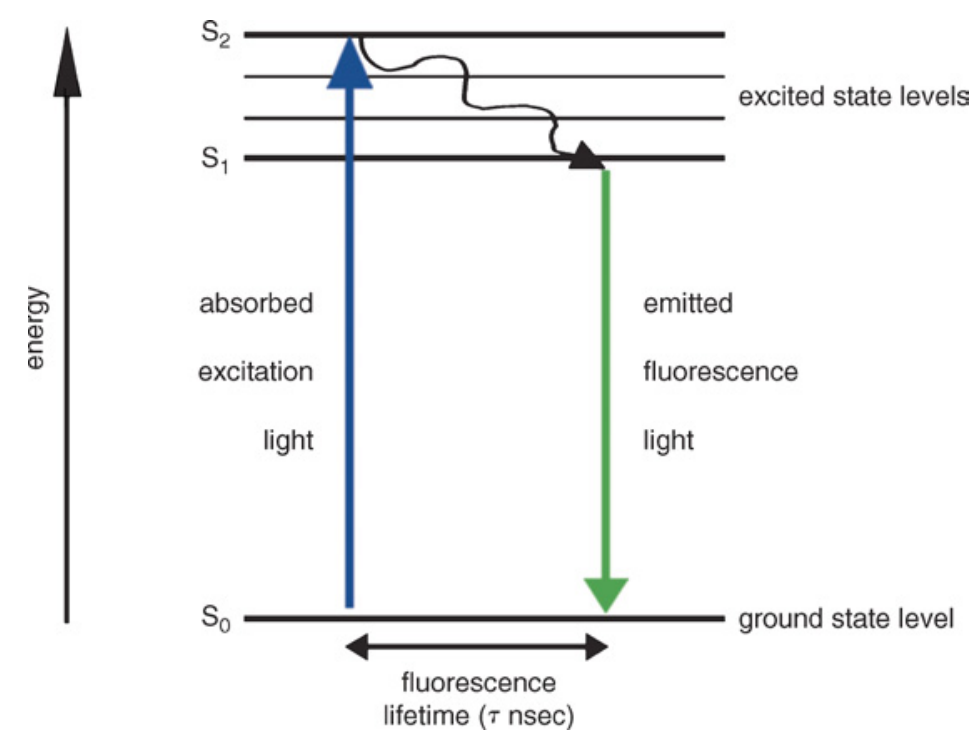

Figure 12.10.1 Fluorescence fundamentals. Jablonski diagram displaying the energy states of a molecule. Molecules not absorbing energy are mainly confined to the ground electronic state level $\mathrm{S}_{0}$. The arrows represent the wavelength of the photons. For example, the arrow to the left (blue in electronic versions of this manuscript) represents the energy of an ultraviolet photon that can cause the molecule to transition from the ground state level $S_{0}$ to either a singlet first electronic excited state $S_{1}$ or to a second electronic excited state $S_{2}$. At each energy level, fluorophores can exist in a number of vibrational energy levels, which are represented by the multiple lines in each electronic state. The electronic transitions are almost instantaneous in nature, often occurring in timeframes ranging from nano- to picoseconds. The arrow on the right (green in electronic versions of the manuscript) represents the lowest energy photon that can be emitted by this molecule as it drops back from the lowest energy excited state of $S_{1}$ to $S_{0}$. The fluorescence lifetime $\left(\tau_{\text {nsec }}\right)$ is the residual time that a molecule, which has absorbed a photon, will remain in the excited state before returning to the ground state level by re-emitting a photon, i.e., fluorescence.

emission is at a longer wavelength (i.e., lower energy) than the light used to excite it (Fig. 12.10.1).

Although the fluorescence of organic molecules can be characterized by their excitation and emission spectra, they can also be characterized by the time it takes for the energized electron to return to the ground state. This is called fluorescence lifetime. When a fluorophore absorbs a photon, it enters an excited state and returns to the ground state by emitting a lower energy photon, with the energy difference between the absorbed and emitted photon transferred to the environment. For almost all fluorophores, the rate of energy transfer from an electron in the excited state is dependent on the local chemical environment, e.g., the concentration of ions, oxygen, and/or pH. It can also be influenced by the binding of proteins in a cell.

One of the many factors that can influence the measured lifetime of a population of fluorophores is the close proximity of a second fluorescent molecule with the appropriate spectral properties to allow it to absorb energy from the first molecule through fluorescence resonance energy transfer (FRET; Lakowicz, 1999). FRET is a very efficient fluorescence quencher. Thus, a decrease in the lifetime of the first (donor) fluorophore can be used to measure a FRET interaction with the second (acceptor) fluorophore (Fig. 12.10.2). However, the transfer of energy between the donor and acceptor fluorophores can only occur when the two molecules are very close together ( $\sim 1$ to $10 \mathrm{~nm}$ ), consistent with being in molecular contact (Lakowicz, 1999; Miyawaki, 2003). Therefore, due to this proximity dependence of FRET, it provides an assay for detecting interactions between two proteins that are coupled with a donor and acceptor FRET

12.10.2 


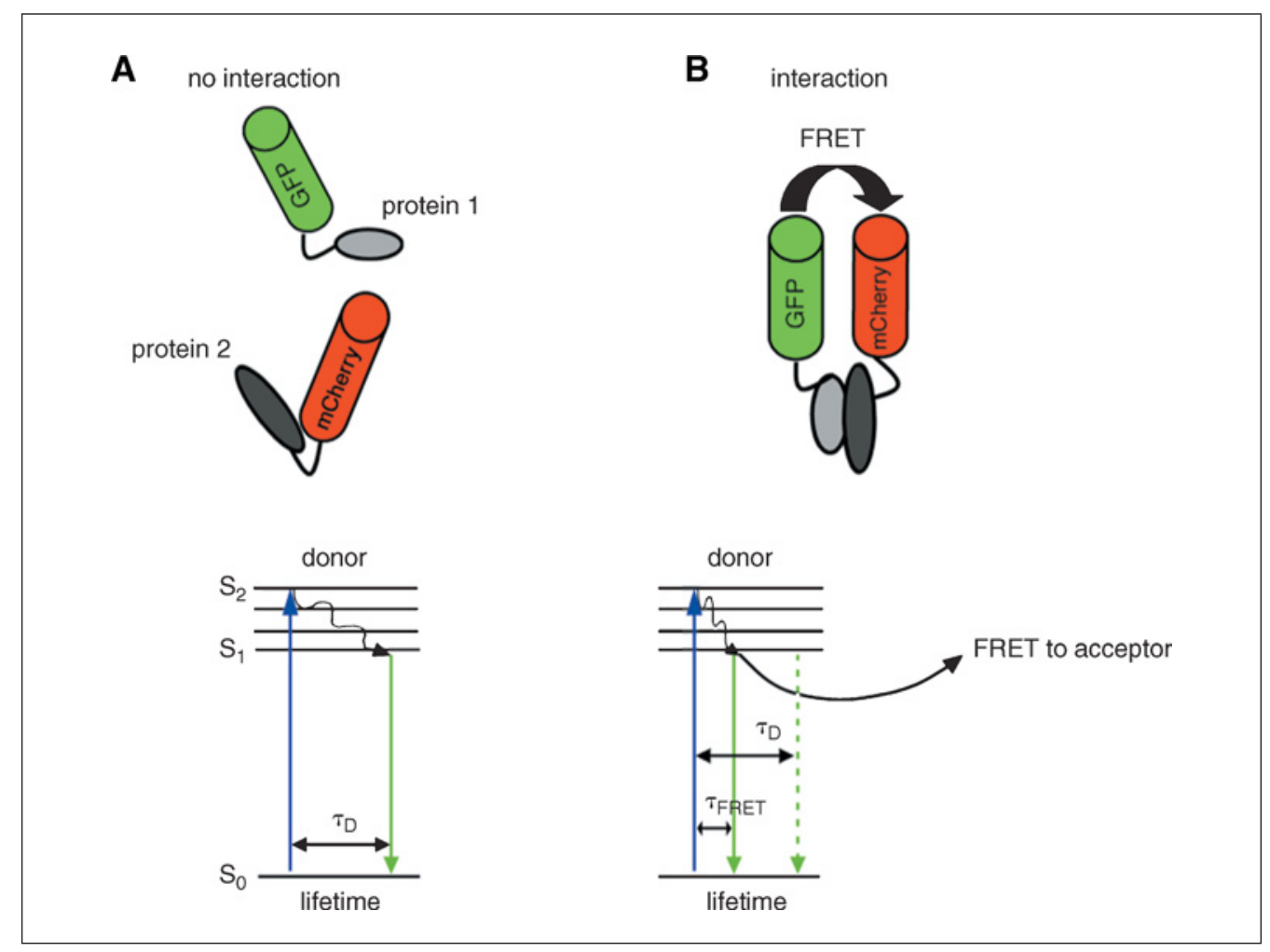

Figure 12.10.2 Detection of fluorescence resonance energy transfer (FRET) by fluorescence lifetime imaging microscopy (FLIM). (A) The two fluorescent fusion proteins do not interact. On absorbing light, the donor fluorophore changes from ground state $\left(\mathrm{S}_{0}\right)$ to the excited state $\left(\mathrm{S}_{2}\right)$, as illustrated in the simplified Jablonski energy-level diagram (A, bottom). This is followed by emission of a photon (fluorescence) during the next few nanoseconds $\left(\tau_{D}\right)$. (B) The two fluorescent fusion proteins interact illustrating the effect of energy transfer on donor fluorescence lifetime. As the Jablonski diagram shows (B, bottom), deactivation from the donor excited state can occur either by fluorescence (downward-pointing arrow), or through the radiationless transfer of energy to the acceptor by FRET. The occurrence of FRET is detectable by a decrease in the donor fluorescence lifetime $\left(\tau_{\mathrm{FRET}}\right)$.

pair. FLIM provides an excellent tool for measuring differences in the lifetime of the fluorescence donor and, hence, for calculating FRET efficiencies.

To carry out FLIM-FRET measurements, the two proteins whose interaction will be studied must each carry a suitable fluorophore. The most convenient way of achieving this, in vivo, is to express both proteins fused to respective fluorescent protein (FP) tags (Fig. 12.10.2A). The two FP tags must display the appropriate spectral properties to allow them to function as a FRET pair. A FLIM-FRET protocol using the fluorophore pair EGFP (enhanced-green fluorescent protein) as a donor and the mCherry (monomeric Cherry red fluorescent variant) as an acceptor (Shaner et al., 2004) is described here. Other combinations of FP-tagged proteins can also be used for FLIM-FRET measurements, such as CFP (cyan fluorescent protein as a donor) and YFP (yellow fluorescent protein as an acceptor). Ideally, the FLIM-FRET procedure should be carried out using cell lines that stably express both FP-tagged proteins, using standard procedures to establish the stably transformed cell lines (Trinkle-Mulcahy et al., 2006). However, because FLIMFRET is generally insensitive to the relative concentrations of the fluorophores, it is also possible to carry out the protocol using cells that are transiently expressing either one or both of the FP-tagged proteins.

NOTE: Refer to APPENDIX $3 B$ for general information on mammalian cell culture.

Cellular and Molecular Imaging

12.10.3 


\section{STRATEGIC PLANNING}

Cultured mammalian cells lines, either stably or transiently expressing the following fusion proteins, are required: (1) EGFP fused to mCherry (positive control), (2) unfused, free EGFP and unfused, free mCherry (negative control no. 1), (3) unfused, free mCherry alone (used for initial imaging calibration of the microscope as described below), (4) protein1-EGFP and unfused, free mCherry (negative control no. 2), and (5) protein1EGFP and protein2-mCherry.

Live cells expressing the respective FP-tagged fusion proteins are imaged on a multiphoton-enabled confocal microscope, such as the Bio-Rad Radiance2100MP, which should be equipped with both an argon ion laser and a green helium neon laser and configured to detect emission from GFP (excitation at $488 \mathrm{~nm}$ ) and mCherry (excitation at 543 $\mathrm{nm})$. FLIM is a photon-counting technique. It is imperative, therefore, that external light (e.g., from LEDs, computer monitors, room lights, etc.) is efficiently excluded. Live-cell imaging of mammalian cells also requires that constant temperature (usually $37^{\circ} \mathrm{C}$ ) is maintained. Live cell chambers that completely enclose the microscope (Fig. 12.10.3) are available (e.g., from Solent UK) and it has been found that these are more effective than chambers that heat only the sample (e.g., Bioptechs FCS2 chamber). These live-cell chambers can be supplied with a matt black coating, which is very efficient at blocking external light from the detectors. If such a chamber is unavailable, the sample should at

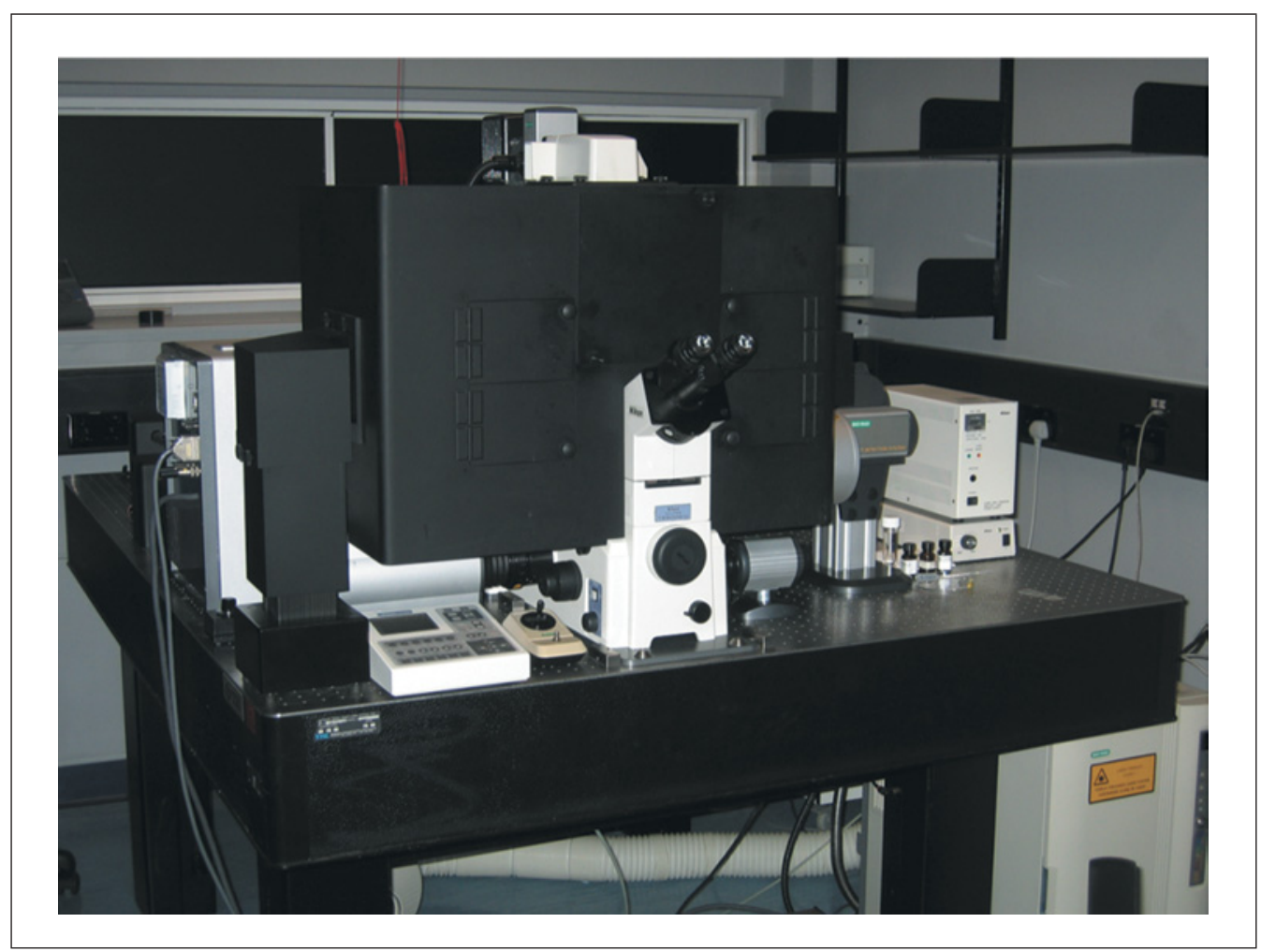

Figure 12.10.3 The multiphoton laser scanning microscope. The Bio-Rad Radiance2100MP multiphoton laser scanning microscope (MPLSM) is equipped with the Coherent Chameleon diodepumped laser (Coherent). The Chameleon laser is a compact, tunable (720 to $930 \mathrm{~nm}$ ), Verdipumped ultrafast laser that produces modelocked, sub-200-fsec pulses at a $90-\mathrm{MHz}$ repetition rate. The microscope is equipped with an environmental chamber suitable to maintain the live cells and optics at constant temperature (usually $37^{\circ} \mathrm{C}$ ). The chamber is constructed with black walls to exclude external sources of light during the sensitive period of FLIM measurement. This system is also equipped with two external detectors (for MPLSM) and two FLIM detectors, which are used with Becker and Hickl's SPC830 board for time-correlated single photon counting (TPSPC) FLIM. 
least be covered with a black box and room lights and monitors should be switched off during acquisition. LEDs can be covered with opaque tape.

The FLIM approach requires an ultrafast, tunable, pulsed laser, such as the Coherent Chameleon Diode-Pumped Laser or the Spectra-Physics Mai Tai, capable of producing femtosecond pulses at a high-repetition rate $(\sim 90 \mathrm{MHz})$.

\section{MEASURING FLIM-FRET IN LIVE MAMMALIAN CELLS}

\section{Materials}

Mammalian cultured cell lines (e.g., HeLa; ATCC \#CCL-2)

Enhanced GFP-C1 (EGFP-C1; Clontech), monomeric Cherry red variant (mCherry-C1; a gift from R.Y. Tsien at http://www.tsienlab.ucsd.edu; also available from Clontech; Shaner et al., 2004)

Dulbecco's Modified Eagle's Medium (DMEM; Invitrogen Life Technologies; also see APPENDIX $3 B$ ) supplemented with $10 \%$ fetal bovine serum (FBS; Invitrogen) and $100 \mathrm{U} / \mathrm{ml}$ penicillin-streptomycin (Invitrogen)

$20 \mathrm{mM}$ HEPES

$\mathrm{CO}_{2}$-independent phenol red-free DMEM medium (Invitrogen)

Effectene transfection reagent (Qiagen)

35-mm glass-bottom dish (e.g., WillCo-dish, Intracel)

35-mm coverslips

Laser scanning confocal microscope (e.g., Bio-Rad Radiance 2100MP or similar system) with:

Argon ion laser (488-nm laser line)

Green HeNe laser (543-nm laser line)

Band-pass emission filter 528/50 for EGFP

570-nm long-pass emission filter for mCherry

Photomultiplier tube (PMTs)

Acquisition software (e.g., LaserSharp2000, Zeiss)

Multiphoton excitation laser, e.g., Coherent Chameleon diode-pumped laser, $720-930 \mathrm{~nm}$, Verdi-pumped ultrafast laser that produces modelocked, sub-200-fsec pulses at $90 \mathrm{MHz}$ repetition rate with an output power of $\sim 1.4 \mathrm{~W}$ at the peak of the tuning curve $(800 \mathrm{~nm})$

Dichroic filter 560 LP

Band-pass emission filter 528/50 for EGFP

Two-channel direct detectors suitable for lifetime imaging e.g., Hammamatsu $5783 \mathrm{P}$

Fluoresence lifetime imaging system consisting of:

Black-walled environmental chamber (e.g., Solent Scientific)

Two-channel direct detectors (e.g., Hammamatsu 5783P) with a fast response for FLIM

TCSPC acquisition card SPCM/SPC830 and software for time-correlated single-photon counting (Becker \& Hickl), or comparable software enabling time-correlated single-photon counting

Imaging software, e.g., SPCImage software (Becker \& Hickl)

\section{Prepare plasmids and cells}

1. To observe interactions between two proteins in living cells using FLIM-FRET, subclone cDNAs encoding the proteins of interest into the required fluorescent protein expression vectors using standard procedures. Obtain plasmids for EGFPprotein 1 (donor) and mCherry-protein 2 (acceptor).

2. Perform control experiments to ensure that the fusions are correctly expressed as full-length proteins, functional (if suitable assay is available), and correctly localized

Cellular and Molecular Imaging

12.10.5 
in the cell. It is also important to ensure that expression of the fusion proteins does not reduce cell viability or promote apoptosis.

These control experiments should include characterization of the size and expression level of the expressed fusion proteins using western blot PAGE gel assays, localization of the fusion protein in cells using fluorescence microscopy and FACS analysis to test for cell cycle effects.

3. Before commencing FLIM-FRET measurements on the proteins under study, test the system using a reliable set of positive and negative controls.

A direct fusion of EGFP and mCherry works well as a positive control (Fig. 12.10.4B). This can be generated by subcloning $m$ Cherry in frame into the multiple cloning site of an EGFP expression vector (or vice versa). The choice of restriction sites will determine the number of amino acids separating the two proteins and, hence, their distance. An EGFP-mCherry fusion separated by 7 amino acids shows high FLIM-FRET efficiency (Fig. 12.10.4B). As a negative control, FLIM-FRET can be measured in cells transfected with plasmid vectors expressing both unfused, free EGFP and unfused, free mCherry (Fig. 12.10.4A).

4. To increase the percentage of cells transiently expressing the fluorescent proteins, cells should be split the day before transfection and allowed to reach $70 \%$ to $90 \%$ confluence.

5. Grow cells in dishes with 35-mm coverslips at the bottom of the dish.

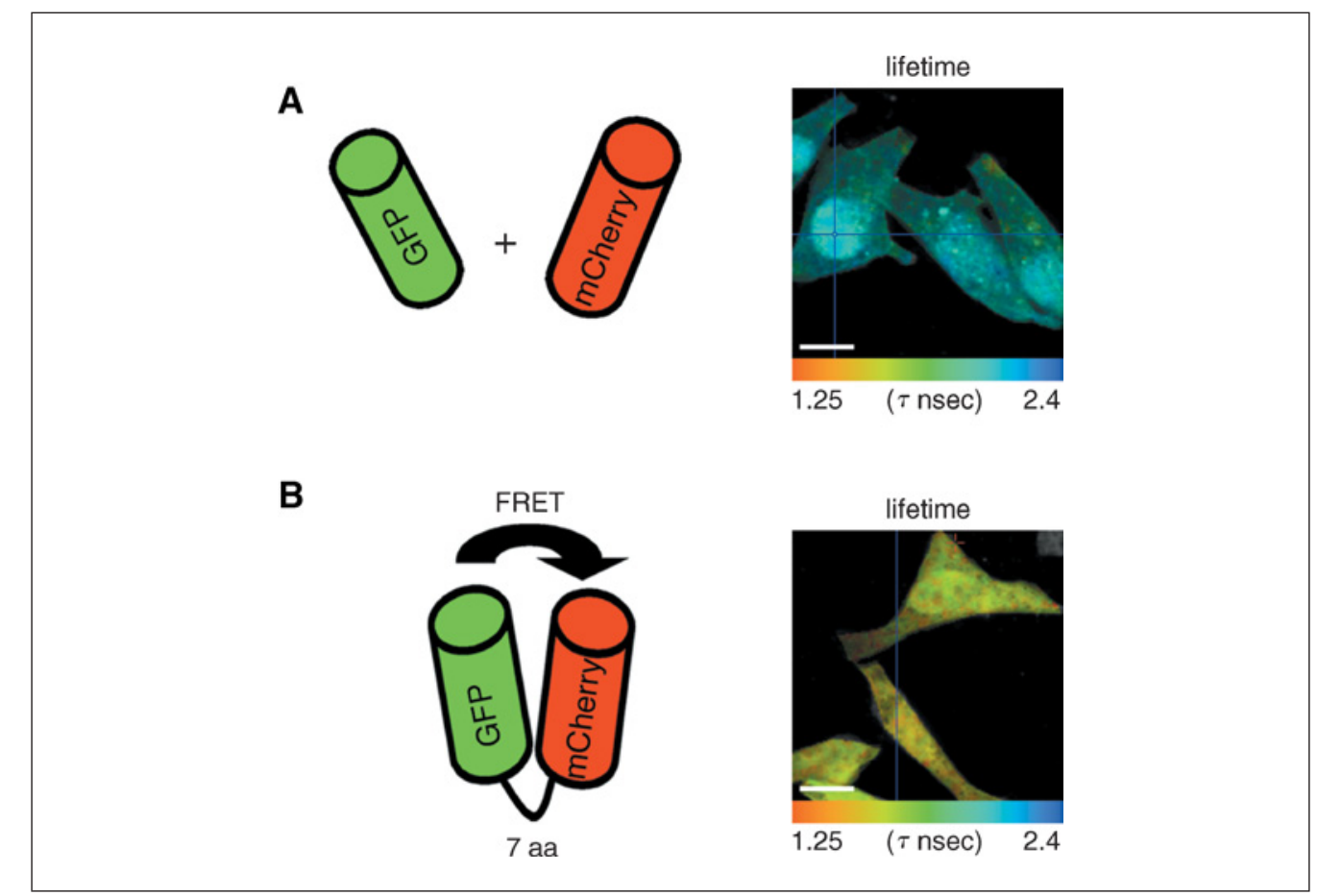

Figure 12.10.4 Evaluation of the accuracy of the FLIM-FRET microscope system by using positive and negative controls. (A) Negative FRET control. Cells co-expressing unfused, free EGFP and unfused, free mCherry, which were co-localized but did not interact, served as a negative FLIM-FRET control. The mean fluorescence lifetime $\left(\tau_{\text {nsec }}\right)$ of the donor GFP was measured using the SPC830 software and a false-colored image displaying the distribution of the lifetime for each pixel of the image is shown. (B) Positive FRET control. Images of cells expressing GFP coupled directly to mCherry through a 7 amino acid linker were acquired and analyzed using the FLIM-

Detecting Protein-Protein Interactions In Vivo with FRET using FLIM
FRET approach. The fluorescence resonance energy transfer from GFP to mcherry leads to a decreased mean fluorescence lifetime for the donor (GFP) from $2.2 \mathrm{nsec}$ to $1.7 \mathrm{nsec}$ throughout the cells. Bars, $10 \mu \mathrm{m}$. For a color version of this figure, see http://www.currentprotocols.com 
6. Transfect cells (Seidman et al., 1997) with the plasmid expression vectors described in steps 1 to 3 to prepare five different experimental conditions:

(a) Control 1: Cells expressing EGFP fused to mCherry (7 amino acid linker)

(b) Control 2: Cells expressing unfused, free EGFP and unfused, free mCherry

(c) Control 3: Cells expressing unfused, free mCherry alone

(d) Control 4: Cells expressing protein1-EGFP and unfused, free mCherry

(e) Control 5: Cells expressing protein1-EGFP and protein2-mCherry.

7. Incubate transfected cells for 14 to $18 \mathrm{hr}$ at $37^{\circ} \mathrm{C}$.

8. For imaging live cells, replace the standard growth medium with phenol red-free medium supplemented with $10 \%$ FBS and $100 \mathrm{U} / \mathrm{ml}$ penicillin-streptomycin. Add $20 \mathrm{mM}$ HEPES to the medium as a buffer. Alternatively, use a custom-made $\mathrm{CO}_{2}$ independent phenol red-free DMEM medium supplemented with FBS and penicillinstreptomycin (this medium does not require additional buffering).

\section{Configure confocal laser scanning microscope for dual GFP/mCherry imaging}

Described below is a three-step process that culminates in the acquisition of lifetime measurements of specific samples, allowing for the calculation of FRET efficiencies, resulting in detection of protein-protein interactions. The steps are: (1) to check that the cells of interest are expressing both the EGFP and mCherry fusion proteins at acceptable levels and showing the correct localization patterns before attempting to measure FRET; (2) to optimize two-photon excitation and imaging parameters; and (3) to optimize lifetime acquisition parameters.

IMPORTANT NOTE: Variants of the fluorescent red protein cannot be excited using standard multi-photon titanium-sapphire wavelengths in the range 720 to $930 \mathrm{~nm}$. mCherry has a two-photon absorption curve beyond the tunable range (peak at $1030 \mathrm{~nm}$ ) of the chameleon laser and, therefore, cannot be imaged directly by two-photon microscopy.

9. Turn on the confocal laser scanning microscope according to the manufacturer's operating instructions.

10. Examine the cells under the microscope and focus on representative fluorescent cells, by centering them in the middle of the field.

11. Check expression levels of fluorescent fusion proteins in the different samples described previously using the confocal laser scanning microscope.

The sample is sequentially scanned using the 488-nm argon ion laser and a 528/50-nm band-pass filter (for EGFP), followed by the $543 \mathrm{~nm}$ HeNe line with a 570-nm long-pass filter (for mCherry).

Start the experiment with the positive control (i.e., cells expressing 7 amino acids separated GFP-mCherry fusion protein (control 1), then repeat with the negative control cells expressing both unfused, free EGFP and unfused, free mCherry (control 2), and repeat again with cells expressing unfused, free $m$ Cherry (control 3 ), then start the experiment by analyzing first cells expressing the donor control, i.e., proteinl-EGFP mixed with unfused, free $m$ Cherry (control 4 ), then finally cells expressing the donor with the acceptor, i.e., protein1-EGFP mixed with protein2-mCherry (control 5).

12. Optimize the image by adjusting the iris, gain, laser power, and offset levels for both GFP and mCherry channels.

IMPORTANT NOTE: Remember the basic compromises in confocal microscopy (UNIT 2.8), i.e., increased gain enhances both signal and noise. Usually, this requires setting the gain and black levels of the photomultiplier detectors together with the pinhole size to obtain the best balance between acceptable resolution and adequate contrast, while

Cellular and Molecular Imaging

12.10.7 
using the lowest laser power possible to minimize photobleaching. Many instruments utilize color look-up tables to aid in setting the correct dynamic range for the image. Such tables are designed so that the darkest pixels, having brightness values around zero, are arbitrarily displayed as green (for example), and the brightest pixels, with brightness values near 255 in an 8-bit system, are displayed as red. The microscope parameters such as gain and black level, and the pinhole diameter, are adjusted so that there are only a few green and red pixels in the image, ensuring that the full dynamic range from 0 to 255 is utilized. For example, increasing the confocal pinhole diameter (iris) also increases the signal reaching the detector, but at the expense of the optical section (Z) resolution. If the aperture is wide open, the equipment is essentially being used as an epifluorescence microscope and no longer as a confocal microscope. Finally, increasing laser power increases the signal, but also increases the amount of light hitting the cells, which can again cause photobleaching and phototoxicity. The use of pseudocolor look-up tables at the extremes of the dynamic range of the detector enables one to set up the imaging system more objectively. These settings will have to be optimized with respect to the nature of the sample. It may be appropriate to open the pinhole wider than usual to keep to a minimum the level of laser light irradiating the sample during the entirety of the experiment.

13. Save the GFP/mCherry confocal method configuration so that these imaging parameters will be ready for future use.

\section{Configure multiphoton scanning microscope for lifetime imaging}

The assay is based on analyzing FRET by the measurement of the fluorescence lifetime of the donor (EGFP). Because the mCherry fluorescent protein cannot be excited by the Chameleon laser, it is not necessary to create an mCherry acquisition setting.

Multiphoton excitation only occurs at the point of focus of the laser beam, thereby generating optical sectioning without the need for an emission aperture (pinhole) as used in confocal laser scanning imaging.

For maximum sensitivity, as much of the emitted fluorescence as possible must be collected. Some of this emitted light will travel directly back to the objective lens, but (increasingly so with depth) a proportion will be scattered on its return path. As it emerges from the back of the objective lens, the scattered light will not be in a parallel beam. To collect as much of this scattered light as possible, the direct detectors are designed with many lenses placed close to the pick-off beam splitter (chromatic reflector), after which a single filter cube splits the emitted light and the light is sent into either one, or both, of the detectors.

14. Turn on the multiphoton laser scanning microscope according to the manufacturer's operating instructions.

15. Create a setting for multiphoton imaging of GFP:

a. From the Optic Configuration Diagram, select the Multiphoton laser as the excitation source. Check that it is modelocked and then set at $890 \mathrm{~nm}$ as the desired excitation laser wavelength to cause two-photon excitation of GFP. Set an appropriate laser power, which can also be adjusted later.

b. Set the light path such that light emitted from the sample is sent to the direct/external detectors (DDS1 position), rather than to the internal detectors (open position) in the confocal light pass.

c. Select the 528/50-nm band-pass filter for GFP.

Detecting Protein-Protein Interactions In Vivo with FRET using FLIM
16. Optimize the image by adjusting the laser power, gain, and offset levels for the GFP channel as described previously for the confocal mode. 
17. Save the GFP_2Photon method configuration (the method will be ready for future use).

\section{Perform fluorescence lifetime imaging microscopy (FLIM) acquisition}

Steps 18 to 27 are to be performed successively with cells expressing the various combinations of control and experimental fusion proteins described above in step 6 .

18. Launch the single photon counting (SPCM) software of the SPC-830 module (Becker \& Hickl).

The time-correlated single photon counting (TCSPC) FLIM system is controlled by the SPCM software of the SPC-830 module (Becker \& Hickl).

Time-correlated single photon counting is based on the detection of single photons of a periodical light signal, the measurement of the detection times of the individual photons and the reconstruction of the waveform from the individual time measurements (Gratton et al., 2003).

The TCSPC technique makes use of the fact that for low-level, high-repetition rate signals the light intensity is usually so low that the probability of detecting one photon in one signal period is much less than one. Therefore, the probability of detecting several photons within one signal period is negligible. The detector signal consists of a train of randomly distributed pulses due to the detection of the individual photons. Consequently, there are many signal periods without any photons, while other signal periods contain one photon pulse. Periods with more than one photon are very rare for the reasons described above. When a photon is detected, the time of the corresponding detector pulse is measured. Each detection event is recorded in memory, associated with its specific detection time. Over time, the waveform of the optical pulse builds up, corresponding to a histogram presenting the number of photons recorded for each detection time interval.

Depending on the desired accuracy, the light intensity must be not higher than required to detect 0.01 to 0.1 photons per signal period. Modern laser light sources, such as the coherent laser described above, deliver pulses with repetition rates of 50 to $100 \mathrm{MHz}$. For these light sources, the count rate constraint is satisfied even at high photon rates of $\sim 10^{6}$ photons/sec (http://www.becker-hickl.com).

19. Use the Predefined Setup Options created during the setup of the SPCM/FLIM system specified by the manufacturer; click on Main, Load Predefined Setups (this opens a secondary panel). A setup is loaded by clicking on the button on the left of the name of the setup. Select the $256 \times 256$ single channel setup.

20. In the upper-most section of the Control panel of the LaserSharp2000 software:

a. Define an image size resolution of $512 \times 512$ pixels.

The laser scanning microscope scans the image area with a constant pixel dwell time, regardless of whether or not the pixels contain useful information. Thus, decrease the acquisition time by excluding empty pixels from being recorded. The simplest way of achieving this is to zoom into the area of the image, which has the least black (i.e., empty) pixels.

b. Set the fastest scan speed (600 lps or $750 \mathrm{lps}$ ) and the same zoom value as defined in step a.

Other frame sizes may also be used; in this case, make sure that the scan control parameters of the SPCM software correspond to that frame size.

21. In the status window of the main panel of the SPCM data acquisition window, the different count rates are displayed as follows:

a. The SYNC (synchronization signal) rate is the repetition rate of the laser. The Ti:Sapphire laser of a Chameleon multiphoton microscope has a repetition rate of

Cellular and Molecular Imaging

12.10.9 
$90 \mathrm{MHz}$. If the SYNC rate differs from the nominal repetition rate of the laser, then either the modelocking is not running properly, or the SYNC threshold is set too high or too low and a service engineer must be consulted.

b. The CFD (constant fraction discriminator), TAC (time to amplitude converter), and ADC (analogue to digital converter) rates indicate the rate of the detected, converted, and stored photons, respectively (Becker, 2005). The rates are direct indicators of the progress of a FLIM measurement. Be aware that several reasons may cause the count rates to fluctuate, e.g., nonhomogeneous intensity in the scan area, beam blanking during the beam flyback, and suppression of photons outside the useful scan area.

The System Parameters panel contains the complete set of measurement control parameters of the TCSPC module. Do not modify these parameters, which instead should be fixed during installation by a qualified engineer.

22. Turn on the laser at a low level (i.e., a few percent of total power), then adjust the Gain and Offset to obtain a CFD and TAC count rate between $5 \times 10^{4}-1 \times 10^{6}$ photons/sec.

Note that higher count rates yield a better signal-to-noise ratio for a given acquisition time.

23. From the previous adjustments, set a defined time of acquisition in the status window of the main panel of the SPCM software.

24. Start a continuous scan in the Lasersharp2000 interface. Start the measurement in the SPCM software by clicking on the Start button. Let the measurement run until it stops, by either (a) completing the selected time of acquisition, or (b) a specified number of accumulation cycles. The resulting image displayed is either (a) the intensity accumulated over all time channels of the pixels, or (b) the intensity within a selected time gate, depending upon the setting selected.

If high laser excitation power is used, it may cause excessive photobleaching of the sample. Photobleaching can change the recorded lifetimes. It is not only the case that different fluorophores (or the same fluorophore in different binding states) bleach at different rates, but also that the photobleaching products may fluoresce themselves. To avoid this, when a FLIM measurement is running, monitor the CFD and TAC count rates. A gradual decrease in the count rates indicates photobleaching. As long as the total decrease does not exceed $\sim 20 \%$, the effect on the lifetimes may still be acceptable. If the decrease is larger than $\sim 20 \%$, either reduce the laser power or increase the detection volume.

25. Finish the measurement by clicking on the Stop button of the SPCM software. Also, separately stop the scan of the LaserSharp2000.

26. Save the data as an .sdt file by using the Save function of the SPCM software.

Only these files contain both photon and setup data. Thus, by saving as an .sdt file, all the metadata information needed to allow the measurement to be reproduced is stored.

27. Send the file to the imaging software SPCImage for data analysis.

\section{Analyze FLIM data for FRET calculations}

Steps 28 to 33 are performed with the data obtained from the respective control and experimental cell lines expressing the various FP fusion plasmids described above.

The data correspond to an array of pixels, with each pixel associated with a histogram showing the number of photons recorded at that pixel for each time interval spanning the lifetime period of the fluorescence decay. Therefore, the FLIM measurement delivers 
images with a separate decay curve for each pixel, allowing FLIM-FRET values not only to be measured but also spatially resolved within the cell; i.e., one obtains a pixel by pixel map of variations in FLIM-FRET, and hence variations in the level of protein-protein interactions, throughout the cell.

To extract the mean fluorescence lifetime for each pixel, the decay curves in the individual pixel must be fitted with an appropriate model, taking into account also the instrument response function (IRF). The IRF is defined as the pulse shape of the FLIM system records for an infinitely short fluorescence lifetime. Typical models are either single exponential, or a sum of exponential terms. The models are normally characterized by several parameters, e.g., the fluorescence lifetimes of the exponential terms and the amplitude of the decay curve (Fig. 12.10.5). The fitting procedure gives these parameters for all pixels of the scan.

In practice, software packages perform these modeling steps automatically to derive the mean lifetime values from the recorded data. Note, however, that there is still a considerable scope for improving this software and the mathematical models used. Thus, future improvements in the algorithms are likely to deliver improvements in the accuracy of the lifetime values that can be derived.

28. Open SPCImage software, and click on File, Import. The import option of SPCImage loads the .sdt files saved by the SPCM data analysis software. A blue cross can be used to select individual pixels, with the resulting intensity image of the loaded FLIM data and corresponding fluorescence decay curve displayed for the selected pixel. If

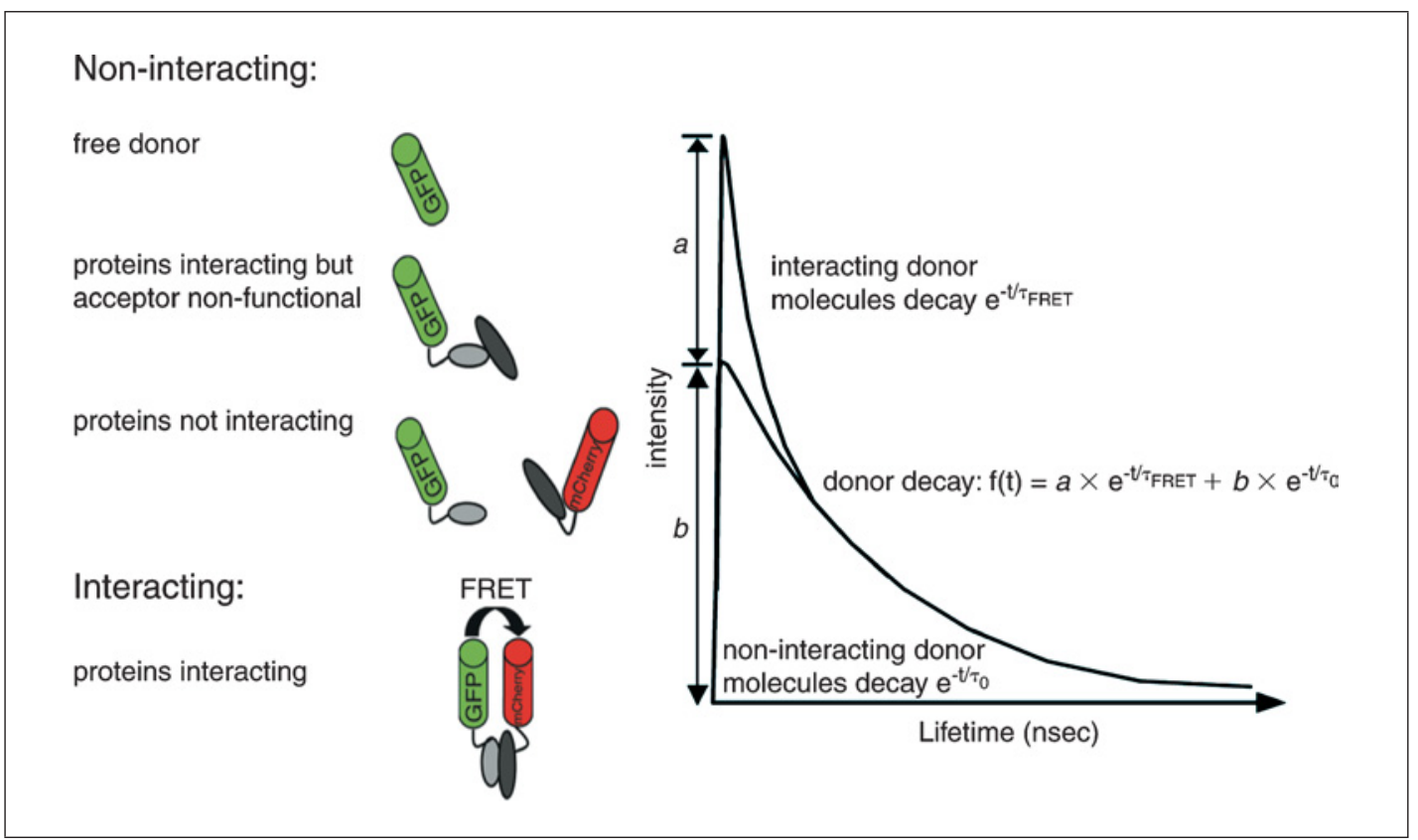

Figure 12.10.5 Fluorescence decay components in FRET conditions. The total decrease of the donor fluorescence intensity, or fluorescence lifetime, depends both on the distance between the donor and acceptor and the fraction of interacting donor molecules. In FRET situations, a double exponential model can approximate the resulting donor decay functions, with a slow lifetime component from the fraction of non-interacting donor molecules and a fast component from the fraction of interacting donor molecules. The composition of the interacting and non-interacting combinations is illustrated on the left of the figure. The composition of the donor decay function is illustrated on the right. Double exponential decay analysis delivers the lifetimes, $\tau_{0}$ and $\tau_{\mathrm{FRET}}$, and the intensity factors, $a$ and $b$, of the two decay components. 
no pixel is selected using the blue cross, the value for the brightest pixel is selected by default.

The hot spot (i.e., brightest pixel) is used to calculate an IRF for the fitting procedure. But, the SPCImage data analysis software estimates an IRF from the recorded data. If desired, however, the IRF can be measured independently before each set of FLIM experiments. To do this, dissolve hydroxyurea in water and let it dry on a microscope coverslip. Seal it with a layer of nail polish, which is allowed to air dry. Scan the coverslip continuously at $920 \mathrm{~nm}$, and directly record the IRF using SPC830 in Oscilloscope mode. Save the data as an .sdt file. Then, from the SPCimage software, click into Load Data, Import, Instrumental response.

29. Define a region of interest (ROI) that will be used during the data analysis by clicking on the white dots and moving them to reduce the area that will be analyzed.

30. In the bottom of the decay panel, select the desired fitting model. To find the appropriate model, perform the following:

a. Select a representative spot in the sample.

b. Increase the Binning factor (defined as the number of pixels around the current pixel position) to optimize the fluorescence decay function.

c. Define a Threshold value by moving the blue cross to a pixel in the background, to establish a minimum number of photons in the peak of a fluorescence curve. Pixels with lower photon numbers are not analyzed by the fitting procedure. This improves the quality of the lifetime parameter histogram.

d. Change the number of exponential components and check the displayed $\chi^{2}$ and the curve of the residuals. A good fit is characterized by a $\chi^{2}$ close to 1 , and residuals showing no noticeable systematic variations. Note that the better the fit, the flatter the residual values. Figure 12.10.6 shows examples that compare good and poor fits to the lifetime decay values.

e. Adjust the Shift parameter to optimize the $\chi^{2}$ value to as close to 1 as possible.

f. The Scatter parameter (amount of scattered excitation light detected) should be adjusted to a value of either zero, or close to zero.

g. Leave the Baseline offset parameter free (i.e., unconstrained).

The best-fitting model can vary and will be influenced by the fluorophores used. For example, a single-exponential model will fit unfused, free EGFP decay curves adequately. In contrast, unfused, free ECFP (enhanced cyan fluorescent protein) presents a more complex decay lifetime and should be fitted using a multi-exponential model (Yasuda et al., 2006).

In the present assay, unfused, free EGFP and unfused, free mCherry (see control 2) can be analyzed using a mono-exponential decay. For the EGFP-mCherry fusion protein positive control (see control 1) or protein1-EGFP and protein2-mCherry ( see control 5), the donor decay should be fitted using a bi-exponential decay model reflecting the fact that there will be both interacting and noninteracting EGFP donor molecules.

31. Choose the decay parameters $\left(\tau_{\mathrm{m}}, \tau_{\mathrm{FRET}}, \mathrm{a}, \mathrm{b}, \mathrm{a} / \mathrm{b}, \mathrm{E}_{\mathrm{FRET}}\right.$, etc. $)$ to analyze by selecting Option, color, then coding value.

32. Start the calculation of the lifetime by selecting Calculate, Decay matrix.

33. Select Option, Color, then continuous or discrete to display a color scale on the lifetime image. 


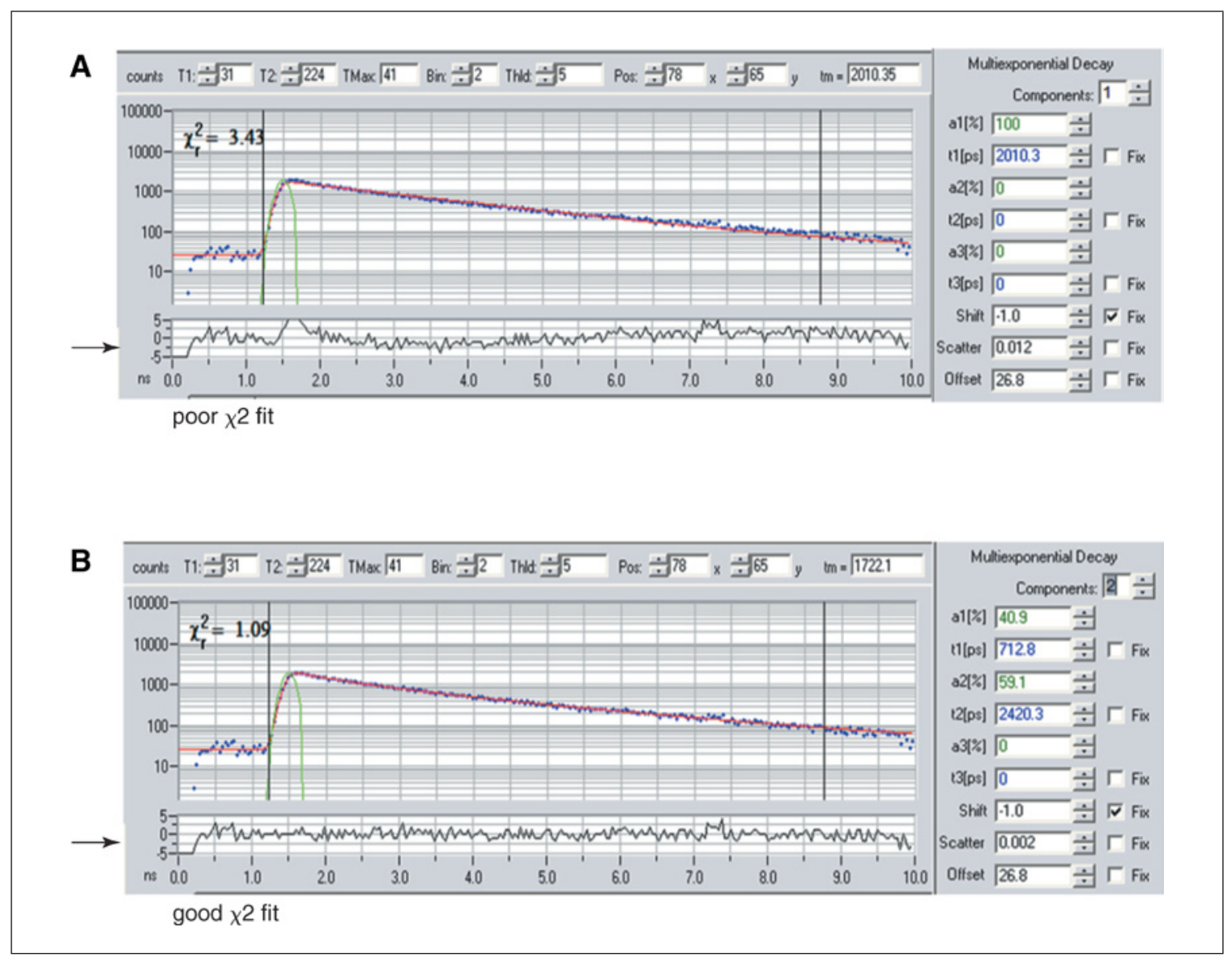

Figure 12.10.6 Comparison of data models. It is often not clear which model, in particular which number of exponential components, should be used to fit the experimental data. In most cases, decay curves can be fitted adequately by a single- $(\mathbf{A})$ or double-exponential model (B). A poor fit can be detected by comparing the fitted curve (red) with the photon data (blue) in the decay window (A). More accurately, a good fit is characterized by a $\chi^{2}$ close to 1 and residuals showing minimal systematic variations, as illustrated in this example (compare the lower variation in the residual values in B with the larger residual fluctuations in A (arrows). For a color version of this figure, see http://www.currentprotocols.com

\section{Calculate FRET}

Steps 34 and 35 below allow calculation of both FRET values and the proportion of interacting proteins, using the FLIM data recorded from the procedure described above. Because FRET interactions cause a decrease in the fluorescence lifetime of the donor molecules (GFP), the FRET efficiency can be calculated by comparing the FLIM values obtained for the GFP donor fluorophore in the presence and absence of the mCherry acceptor fluorophore. This corresponds to FLIM values in cells expressing either EGFPprotein 1 with mCherry-protein2 (control 5), or only EGFP-protein1 with unfused, free mCherry (control 2).

34. First, perform a quick check for evidence of FRET in cells expressing the respective FP-tagged fusion proteins by calculating the respective lifetime values using a monoexponential decay model. The resulting single exponential lifetime image allows one to locate any areas inside the cells where a reduction in the mean lifetime values $\left(\tau_{\mathrm{m}}\right)$ occurs. Such areas of reduced lifetime correspond to sites where protein1-EGFP interacts with protein2-mCherry.

A reduction in the measured lifetime, as determined using the single exponential function, can be used not only to infer the presence of FRET but also to determine its locations within the cell. However, to extract robust quantitative information regarding parameters such as the mean distance between the interacting proteins and the fraction of the donor protein participating in the protein-protein interaction, it is necessary to analyze the data again using a bi-exponential function (Fig. 12.10.5).

Cellular and Molecular Imaging

12.10.13 
35. The bi-exponential fluorescence decay model provides information about the lifetimes of two populations of molecules, i.e., the non-interacting donor population (lifetime $\tau$ ) and the donor population that is interacting with the acceptor (lifetime $\tau_{\text {FRET }}$ ), as well as the intensity factors, $a$ and $b$, of the two decay components (Figs. 12.10.5 and 12.10.6). From these parameters, the FRET efficiency, ERRET, can be derived from the following equation:

$$
\mathrm{E}_{\mathrm{FRET}}=1-\left(\tau_{\mathrm{FRET}} / \tau\right)
$$

The ratio of the interacting and noninteracting donor molecules $\left(\mathrm{N}_{\mathrm{FRET}} / \mathrm{N}_{0}\right)$ corresponds to the ratio $a / b$.

Finally, the distance $(r)$ between the two interacting proteins can be estimated by the following equation:

$$
r=\mathrm{R}_{0} \times\left[\left(1 / \mathrm{E}_{\mathrm{FRET}}\right)-1\right]^{1 / 6}
$$

where $\mathrm{R}_{0}$ is the Forster distance at which $50 \%$ energy transfer takes place. In the case of the donor-acceptor EGFP/mCherry FRET pair, the value of $\mathrm{R}_{0}$ has been calculated as $4.7 \mathrm{~nm}$ (Tramier et al., 2006).

The above calculations are made without adjusting any of the variable parameters (e.g., $a, b, \tau$, and $\left.\tau_{F R E T}\right)$. However, in some cases, it may be helpful to assume that one of these parameters, such as the lifetime of the noninteracting donor population $(\tau)$, remains constant. In this case, the value of $\tau$ can be obtained by measuring the GFP lifetime in the absence of FRET (i.e., from cells expressing EGFP-proteinl and unfused, free mCherry), and this value used in all subsequent calculations. From the authors' experience of analyzing $\tau$ lifetime values for EGFP fusion proteins, it is reasonable to assume that $\tau$ does not vary significantly from pixel to pixel for different subcellular compartments, the main exception being within lysosomes, where $\tau$ is altered due to the low $\mathrm{pH}$.

\section{COMMENTARY}

\section{Background Information}

\section{FRET basics}

Protein-protein interactions are critical for many processes in mammalian cells. Such interactions include the stable association of proteins within multi-subunit complexes and the transient association of regulatory proteins. Information about such protein-protein interactions in mammalian cells has previously come mostly from in vitro analyses, especially using recombinant, bacterially expressed proteins. A limitation of this approach is that the protein-protein interaction is usually studied in isolation, without regard to competing protein interactions that can occur in the cell and without addressing the spatial organization of such interactions in vivo. Similarly, yeast 2hybrid studies address whether mammalian proteins are capable of interacting, without revealing whether they actually are involved in such interactions under physiological conditions. A major advantage of the FLIM-FRET technique presented here is that it both detects and spatially resolves protein-protein interactions within the context of a living mammalian cell.
It is important to remember the limitations of light microscopy in regards to spatial resolution. Thus, the simple colocalization of two proteins in the same subcellular location does not provide evidence that they interact. The resolution of the light microscope is limited to $\sim 250 \mathrm{~nm}$ laterally and $\sim 500 \mathrm{~nm}$ axially and, therefore, can at best only indicate that the two proteins are located within the same $\sim 250 \times$ $250 \times 500 \mathrm{~nm}^{3}$ volume element (voxel). Because typical globular proteins are $\sim 5$ to $10 \mathrm{~nm}$ in diameter, many such molecules can reside within the same voxel but not directly interact. To provide evidence of protein-protein interaction, it is therefore essential to measure the proximity of proteins with a resolution of $\sim 1$ to $10 \mathrm{~nm}$, which is simply not possible by colocalization using conventional fluorescence microscopy.

This limitation imposed by the resolution of visible light can be overcome by the FRET technique (Herman, 1989). FRET is based on the ability of a donor fluorophore to transfer some of the energy from its excited state to an adjacent acceptor fluorophore, thereby reducing the amount of energy that the donor 
releases as fluorescence. Because the FRET energy transfer is highly distance-dependent, detection of FRET requires that the two fluorophores must be within $\sim 1$ to $10 \mathrm{~nm}$, i.e., the distance typically found for directly interacting proteins.

For FRET to occur, the donor fluorophore must have an emission spectrum overlapping the excitation spectrum of the acceptor fluorophore. In addition, FRET requires that the two fluorophores are both in close proximity and correctly oriented for resonance energy transfer to occur. The FRET efficiency, $\mathrm{E}_{\mathrm{FRET}}$, is given by $\mathrm{E}_{\mathrm{FRET}}=1 /\left[1+\left(r / \mathrm{R}_{0}\right)^{6}\right]$, where $r$ is the distance between the two fluorophores and $\mathrm{R}_{0}$ is the distance at which $50 \%$ energy transfer takes place. The $\mathrm{R}_{0}$ value varies for different fluorophores and is dependent on several parameters, including the amount of overlap between the excitation and emission spectra of the donor and acceptor, the relative orientation of the donor and acceptor and the quantum yield, or brightness, of the donor (Patterson et al., 2000). Due to the sixth-power dependence on the spatial separation, $r$, the FRET efficiency increases dramatically when $r$ is below $\sim \mathrm{R}_{0}$. Thus, FRET detects the apposition of fluorophores specifically when they are within $\sim 1$ to $10 \mathrm{~nm}$.

\section{Techniques for FRET measurement}

There are three commonly used light microscopy approaches for detecting FRET: (1) sensitized emission, (2) acceptor photobleaching, and (3) FLIM (fluorescence lifetime imaging microscopy).

To measure FRET by sensitized emission, the donor fluorophore is excited and the resulting increased fluorescence of the acceptor, caused by transfer of energy from the donor, is detected (Trinkle-Mulcahy et al., 2006). This method can be performed using more or less any fluorescence microscopes with highquality filters and a sensitive detector, e.g., either a laser scanning confocal fluorescence microscope, or a wide-field fluorescence microscope. However, while it is technically the simplest assay to perform, a major limitation of using sensitized emission to measure FRET is the complicated correction and normalization procedures that are required to interpret the data. These arise from the fact that three independent processes contribute to fluorescence in the acceptor emission channel: (1) fluorescence of the acceptor as a result of FRET, (2) fluorescence of the acceptor induced by light intended to excite the donor, and (3) spectral bleed-through of donor emission into the ac- ceptor emission channel. Thus, the measurements are strongly influenced by the relative concentrations of the respective fluorophores, which can be difficult to control.

The second approach for measuring FRET, acceptor photobleaching, is based on the fact that FRET reduces the amount of fluorescence released by the donor fluorophore (Karpova et al., 2003; Chusainow et al., 2005). Therefore, there is an increase in the donor fluorescence signal that can be measured when FRET is disrupted by photobleaching of the acceptor fluorophore. This approach requires the cells to be illuminated with a suitable laser line at high intensity to completely photobleach the acceptor and the use of appropriate excitation and emission filter sets to monitor donor fluorescence before and after the bleach event. Acceptor photobleaching can be done using either a confocal laser scanning fluorescence microscope, or a wide-field fluorescence microscope equipped with an external laser, such as the DeltaVision Spectris system. This is often a more reliable method than sensitized emission because only the donor channel fluorescence is measured. Therefore, bleed-through from the acceptor is negligible under normal conditions and virtually nonexistent after photobleaching, thereby avoiding the need for the corrections in the data analysis associated with sensitized emission. However, it is also not without technical complications. For example, protein diffusion can be a problem if the acceptor protein diffuses rapidly. This is because the unquenched donor signal will only be observed at very early time-points after bleaching, which can require faster imaging speed than can be achieved with some confocal laser scanning fluorescence microscopes (CLSM) and certain CCD cameras. Another limitation of the acceptor photobleaching approach is that it typically can be applied only once in a live cell due to the effects of protein diffusion. Also, it generally only measures FRET at a single predetermined location, rather than at every pixel throughout the cell. As a result, acceptor photobleaching is not usually suitable for either time-lapse FRET measurements, or for detailed mapping of FRET locations at different sites within a cell.

In the third approach, FRET is measured by FLIM (Wallrabe and Periasamy, 2005). This provides the most reliable and quantitative approach for FRET detection, but also requires the most complicated and expensive instrumentation. The FLIM-FRET approach is based on measuring changes in the fluorescence lifetime of the donor molecule as a result
Cellular and Molecular Imaging

12.10.15 
of FRET interactions; this method is described in detail in this unit. The major advantages of using FLIM-FRET include the fact that it can provide a unique measurement of the amount of FRET occurring for every pixel in an image and is not affected significantly by either the relative concentrations of the interacting proteins, or by their diffusion rates. FLIM-FRET can also provide additional information about the fraction of proteins engaged in interactions and their mean separation distance (Yasuda et al., 2006)

FRET is a powerful approach for identifying the presence of protein-protein interactions within the context of an intact cell. Whichever method is used to detect and measure FRET, interactions are studied in a more physiological background and at least using FLIM-FRET such interactions can also be resolved in space and time. It is important to note, however, that the precise molecular requirements for establishing a situation that produces resonance energy transfer are not always met, even when two proteins do interact. For example, either the orientation of the respective fluorophores may be incorrect, or the folding of the proteins may move the fluorophores sufficiently far apart that little or no FRET occurs. Therefore, while the presence of FRET can reasonably be taken as evidence of protein-protein interaction, the absence of FRET cannot be safely interpreted as meaning that the two proteins under study are not interacting. It can be helpful, in some cases, to search for FRET interactions between proteins believed to interact by moving the fluorescent tags between the amino and carboxy termini of the respective fusion proteins. In the authors' opinion, it is useful to apply the FRET approach in conjunction with other methods for examining and characterizing protein-protein interactions, including in vitro techniques using isolated fusion proteins.

\section{Critical Parameters and Troubleshooting}

\section{Fluorophores}

Because fluorescence lifetime is highly sensitive to environmental factors, such as $\mathrm{pH}$ and temperature etc., it is essential to control, as much as possible, the external factors that can affect the lifetime value of the donor fluorophore during FLIM-FRET measurements. These controls will help to discriminate between a decrease in donor lifetime resulting from FRET as opposed to other variations in lifetime caused by the fluctuations of external parameters leading to non-FRET relaxation. Therefore, care should be taken to execute the FLIM measurements at a constant temperature and $\mathrm{pH}$. It is also essential to perform the free donor control measurement (i.e., protein1GFP with unfused, free mCherry) using exactly the same experimental conditions that are used to measure the protein1-GFP with protein2-mCherry (i.e., donor plus acceptor). This is especially important to remember if conditions have been intentionally changed as part of the experiment, for example, treating cells with inhibitors or inducing heat-shock stress.

Before the GFP era, FRET required either direct labeling of proteins with fluorophores to serve as donor and acceptor, or indirect labeling of the interacting proteins using antibodies conjugated to the donor and acceptor fluorophores. Direct labeling is a laborious procedure that requires protein isolation, dye conjugation, purification, and then subsequent reintroduction into the cell. Each of these steps can prove technically difficult both to perform and to control. Indirect antibody labeling is considerably simpler, but nonspecific binding can cause problems and the large size of the antibody molecules can result in the donor and acceptor fluorophores being too far apart for energy transfer to take place. The advantages of the GFP-fusion technology for measuring FRET are (1) the direct labeling of the proteins of interest, (2) the relative simplicity of constructing the GFP fusions and introducing them into cells, and (3) the ability to perform FRET measurements and thereby examine the dynamics of protein-protein interactions in live cells.

The advent of GFP derivatives suitable for FRET has stimulated a resurgence of interest in this technique. However, the choice of the fluorophore pairs is also crucial for the design of FLIM-FRET experiments. Indeed, it is necessary to keep in mind that sensors for fluorescence lifetime measurements have different design criteria than those for intensity based FRET methods, such as sensitized fluorescence or acceptor photobleaching. Although it is still necessary for the donor fluorophore to have an emission spectrum overlapping the excitation spectrum of the acceptor fluorophore, it is also highly recommended that the chosen donor fluorophore have a single-exponential fluorescence decay to facilitate subsequent calculation of the fraction of interacting proteins. Enhanced green fluorescent protein (EGFP) and Venus (the brighter variant of enhanced yellow fluorescent protein, EYFP) have high 
quantum yields and have fluorescence lifetime single exponential decay curves (Nagai et al., 2002), unlike either enhanced cyan fluorescent protein (ECFP), or its brighter variant Cerulean (Rizzo et al., 2004). In the authors' experience, using two-photon excitation EGFP is brighter than EYFP, therefore, EGFP was used as a donor. A monomeric variant of EGFP, (mEGFP, corresponding to EGFP A206K) can also be used as a donor (Zacharias et al., 2002). Based on the authors' experimental data, dimerization of EGFP did not result in complex decay curves for the donor lifetime. Thus, it is not essential to use the mEGFP mutant as a donor.

For fluorescence lifetime measurements, the acceptor quantum yield is not critical because only the donor fluorescence is followed. Acceptors with low quantum yield may even be preferable because this will minimize spectral bleed-through. From the authors' experience, the red fluorescent protein mCherry is an excellent acceptor showing negligible spectral bleed-through (Yasuda et al., 2006).

Some disadvantages should also be considered when using fluorescent fusion proteins. Typically, for any fluorescent fusion protein, distinct populations with and without a functional chromophore coexist in a cell (Nagai et al., 2002). This includes proteins where the FP tag is misfolded, and therefore, either not fluorescent or misoriented, as well as the pool of endogenous, untagged proteins that usually are present in mammalian cells because the experimental design involves co-expression of the tagged form rather than gene replacement. Because interacting proteins without a functional chromophore do not contribute to FRET, they reduce the FRET signal and lead to an underestimation of the fraction of interacting proteins. The fraction of mCherry without a functional chromophore can be deduced from the EGFP fluorescence decay curves of cells expressing the positive control FRET fusion protein, i.e., EGFPmCherry with a 7-amino-acid linker (see Fig. 12.10.4). From previous data using this positive control, FLIM-FRET analysis indicated that the EGFP-mCherry FRET pair had a maximum effective binding fraction of $\sim 0.50$ (data not shown). Quantitative measurements of the fraction of interacting proteins in an experiment must take this factor into account. Furthermore, it has been observed that the maturation of the mCherry fluorophore is slower than the corresponding maturation of EGFP, especially within 28-hr post-transfection (data not shown). This can also cause an underes- timation of the fraction of proteins interacting, as derived from the FLIM-FRET data. To take this into account, measuring FLIM-FRET at multiple time points after transfection and noting when the plateau value is reached is recommended.

\section{Acquisition time, photobleaching, and photon-count rates}

A compromise must be found between factors that influence the FRET measurement, i.e., the choice of the laser power (which causes bleaching of the fluorophore if too intense), and the acquisition time (which allows enough photons to be collected for accurate statistical analysis). The balance is between being able to collect enough emitted photons to extract a representative fluorescence lifetime value from the sample without bleaching the donor fluorophores during the acquisition time (or killing the cell).

Typical FLIM samples are characterized by low fluorophore concentrations. Moreover, most of the effects investigated by FLIM have to be measured in the context of living cells or tissue, which can have low photostability. In practice, reducing the laser excitation power and compensating for the lower count rate by an increased acquisition time can often reduce photobleaching.

It is often stated that photobleaching is lower for two-photon excitation than for onephoton excitation. This is not entirely correct. Of course, two-photon excitation does not cause excitation outside the focal volume, and, consequently, little or no photobleaching occurs above and below the focal plane. However, for the same number of emitted photons, photobleaching within the scanned plane is stronger (Dittrich and Schwille, 2001). It is difficult to provide general recommendations for adjusting the count rate. On the one hand, the concentration of the fluorophore and its photostability may vary by orders of magnitude; on the other hand, the consequence of possible negative effects caused by photobleaching may differ considerably depending upon the design of specific experiments. The authors recommend that the operator is simply aware of these potential effects and considers their possible impact when designing their experiments. In most published FLIM studies, the effect of count rates and the resulting amount of photobleaching are rarely mentioned or considered.

One of the most critical parameters in a FLIM experiment is the need to record a large number of photons. Moreover, the
Cellular and Molecular Imaging

12.10.17 
required number of photons increases if multiexponential decay functions are to be resolved. Some previous studies estimated that a value of $\sim 400,000$ photons must be collected to resolve differences between two separate lifetimes with closely spaced values (Köllner and Wolfrum, 1992). It is often impractical, especially for living cells, to collect such a large number of photons within a reasonable time period. Fortunately, FRET measurements usually involve sufficiently large changes in a lifetime that fewer photons per pixel can be collected for analysis. In general, optimize the time of photon collecting to balance the number of photons required for accurate analysis with the photostability of the sample. It is also important to note that the collection time is the single biggest drawback of time-domain FLIM (TCSPC) for fluorescence lifetime measurements as opposed to frequency domain (phase and modulation) methods (Lakowicz, 1999). Therefore, time-domain (TCSPC) methods are ideal for imaging relatively slow (>30 sec) dynamic processes in small compartments (Gratton et al., 2003).

\section{Anticipated Results}

An example of a FLIM-FRET experiment is shown in Figure 12.10.7. In this case, the change in fluorescence lifetime values resulting from altering the length of the linker peptide separating GFP and mCherry in the positive control fusion protein is demonstrated. Thus, a linker of 7 amino acids shows a larger change in the mean lifetime $\left(\tau_{\mathrm{m}}\right)$, from 2.34 nsec to $2.05 \mathrm{nsec}$ (Fig. 12.10.7A,C), as compared with a linker of 17 amino acids (Fig. 12.10.7A,B). Additionally, the characteristic lifetime associated exclusively with the fraction of interacting donor protein $\left(\tau_{\text {FRET }}\right)$ can be resolved and shifts from $2.34 \mathrm{nsec}$ to $1.58 \mathrm{nsec}$ in the case of the 7-amino-acid linker positive control fusion protein (Fig. 12.10.7A,C). This value can be used to calculate the relative percentage of FRET efficiency. These differences result largely from the increased distance, as

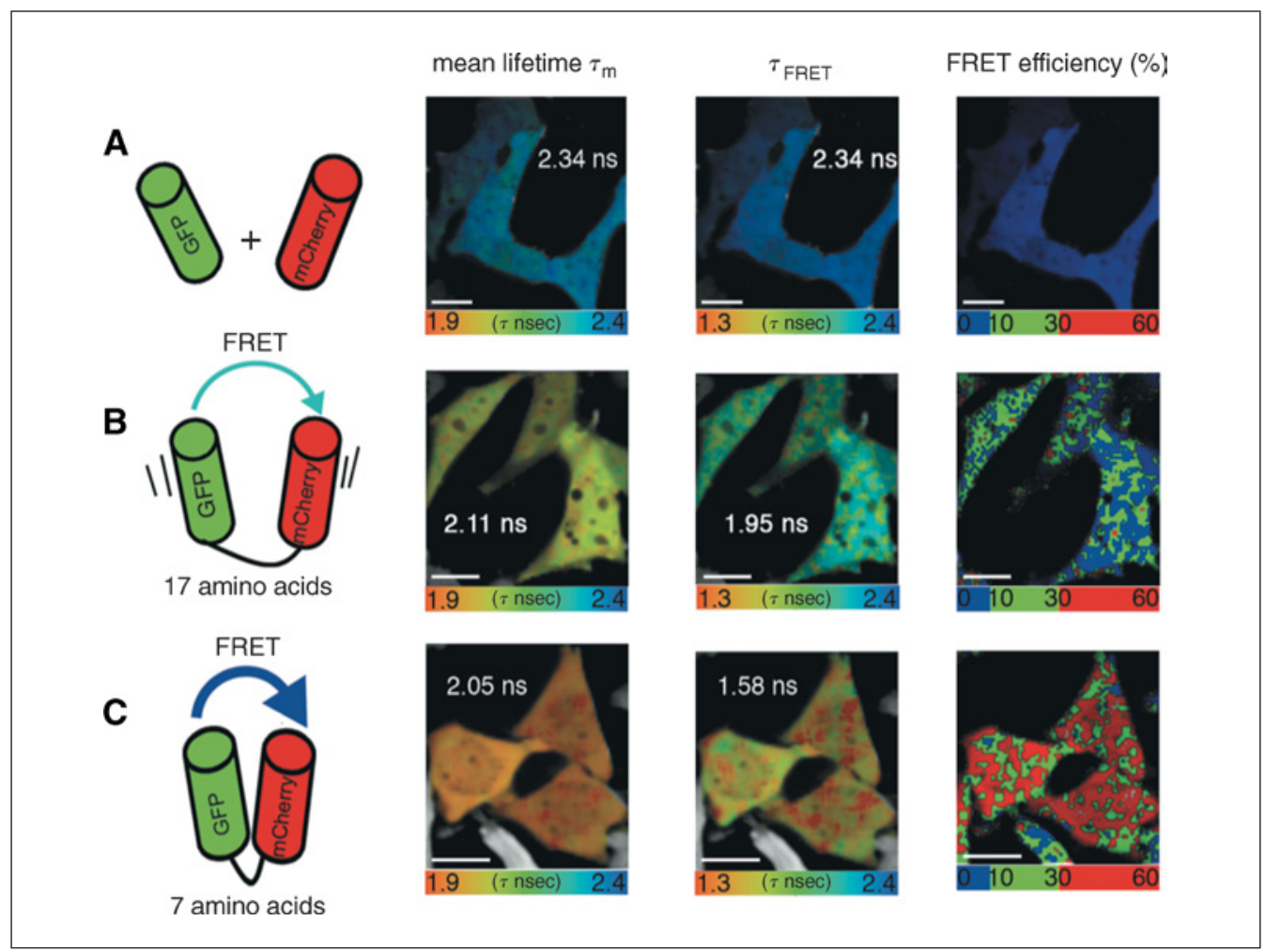

Figure 12.10.7 In vivo FLIM-FRET measurements. Living HeLa cells co-expressing either unfused, free EGFP and unfused, free mCherry (A), or GFP-coupled directly to mCherry through a 17-amino-acid linker (B), or GFP-coupled directly to mCherry through a 7-amino-acid linker (C) were imaged by using the multiphoton scanning microscope. The fluorescence lifetime was analyzed by using the SPCImage software. For each panel, the spatial distribution of the mean fluorescence lifetime $\left(\tau_{\mathrm{m}}\right)$ and of the fluorescence lifetime of the donor molecules interacting with the acceptor $\left(\tau_{\mathrm{FRET}}\right)$ is shown throughout the cells. The FRET efficiencies were calculated for each pixel as $\mathrm{E}_{\mathrm{FRET}}(\%)=\left(1-\tau_{\mathrm{FRET}} / \tau_{\mathrm{D}}\right) \times 100$. Color scale shown covers the range of $\mathrm{E}_{\mathrm{FRET}}$ values from $0 \%$ to $60 \%$. Bars, $10 \mu \mathrm{m}$. For a color version of this figure, see http://www.currentprotocols.com 
well as the alteration in geometry, between the donor and acceptor fluorophores in the two fusion proteins.

\section{Time Considerations}

Initially, there is a significant time requirement to perform the required control experiments and optimize the instrument setup and experimental design for FLIM-FRET studies. Leaving aside the time required to construct reagents, including cell lines and FP-fusion proteins, etc., this set-up time can easily involve several weeks of work, depending upon the experience of the operator. After setting up the experiment, the final imaging time for one acquisition of FLIM-FRET data will typically take less than $\sim 5 \mathrm{~min}$. This can vary significantly depending on the imaging equipment and sample being analyzed. The total collection time also depends upon how many images are analyzed for each experimental condition. To obtain robust data, analyze at least 20 cells independently in each experiment. This will require a total imaging period of $\sim 5 \mathrm{hr}$ to carry out the whole experiment. Subsequently, analysis of the data recorded above using the SPCImage analysis software might take an additional $5 \mathrm{hr}$. It is advisable to perform at least three independent repetitions of each entire experiment.

\section{Acknowledgements}

The authors thank Dr. Silvana van Koningsbruggen for helpful discussions and suggestions. The authors are grateful to Dr. Laura Trinkle-Mulcahy, Dr. Paul Appleton, and Calum Thomson for their advice and support. A.I. Lamond is a Wellcome Trust Principal Research Fellow.

\section{Literature Cited}

Becker, W. 2005. The BH TCSPC Handbook, Becker and Hickl GmbH.

Chusainow, J., Ajuh, P.M., Trinkle-Mulcahy, L., Sleeman, J.E., Ellenberg, J., and Lamond, A.I. 2005. FRET analyses of the U2AF complex localize the U2AF35/U2AF65 interaction in vivo and reveal a novel self-interaction of U2AF35. RNA 11:1201-1214.

Dittrich, P.S. and Schwille, P. 2001. Photobleaching and stabilization of fluorophores used for singlemolecule analysis with one- and two-photon excitation. Appl. Phys. B 73:829-837.

Gratton, E., Breusegem, S., Sutin, J., Ruan, Q., and Barry, N. 2003. Fluorescence lifetime imaging for the two-photon microscope: Time-domain and frequency-domain methods. J. Biomed. Opt. 8:381-390.
Herman, B. 1989. Resonance energy transfer microscopy. Methods Cell Biol. 30:219-243.

Karpova, T.S., Baumann, C.T., He, L., Wu, X., Grammer, A., Lipsky, P., Hager, G.L., and McNally, J.G. 2003. Fluorescence resonance energy transfer from cyan to yellow fluorescent protein detected by acceptor photobleaching using confocal microscopy and a single laser. $J$. Microsc. 209:56-70.

Köllner, M. and Wolfrum, J. 1992. How many photons are necessary for fluorescence-lifetime measurements? Phys. Chem. Lett. 200:199-204.

Lakowicz, J.R. 1999. Principles of Fluorescence Spectrocopy 2nd editon. Springer, New York.

Miyawaki, A. 2003. Visualization of the spatial and temporal dynamics of intracellular signaling. Dev. Cell 4:295-305.

Nagai, T., Ibata, K., Park, E.S., Kubota, M., Mikoshiba, K., and Miyawaki, A. 2002. A variant of yellow fluorescent protein with fast and efficient maturation for cell-biological applications. Nat. Biotechnol. 20:87-90.

Patterson, G.H., Piston, D.W., and Barisas, B.G. 2000. Forster distances between green fluorescent protein pairs. Anal. Biochem. 284:438-440.

Rizzo, M.A., Springer, G.H., Granada, B., and Piston, D.W. 2004. An improved cyan fluorescent protein variant useful for FRET. Nat. Biotechnol. 22:445-449.

Seidman, C.E., Struhl, K., Sheen, J., and Jessen, T. 1997. Introduction of plasmid DNA into cells. Curr. Protoc. Mol. Bio. 37:1.8.1-1.8.10.

Shaner, N.C., Campbell, R.E., Steinbach, P.A., Giepmans, B.N., Palmer, A.E., and Tsien, R.Y. 2004.Improved monomeric red, orange and yellow fluorescent proteins derived from Discosoma $s p$. red fluorescent protein. Nat. Biotechnol.22:1567-1572.

Tramier, M., Zahid, M., Mevel, J.C., Masse, M.J., and Coppey-Moisan, M. 2006. Sensitivity of CFP/YFP and GFP/mCherry pairs to donor photobleaching on FRET determination by fluorescence lifetime imaging microscopy in living cells. Microsc. Res. Tech. 69:933-939.

Trinkle-Mulcahy, L., Chusainow, J., Lam, Y.W., Swift, S., and Lamond, A. 2006. Visualization of intracellular PP1 targeting through transiently and stably expressed fluorescent protein fusions. Methods Mol. Biol. 365:133-154.

Wallrabe, H. and Periasamy, A. 2005. Imaging protein molecules using FRET and FLIM microscopy. Curr. Opin. Biotechnol. 16:19-27.

Yasuda, R., Harvey, C.D., Zhong, H., Sobczyk, A., van Aelst, L., and Svoboda, K. 2006. Supersensitive Ras activation in dendrites and spines revealed by two-photon fluorescence lifetime imaging. Nat. Neurosci. 9:283-291.

Zacharias, D.A., Violin, J.D., Newton, A.C., and Tsien, R.Y. 2002. Partitioning of lipid-modified monomeric GFPs into membrane microdomains of live cells. Science 296:913-916.
Cellular and Molecular Imaging

12.10.19 